\title{
Effects of cavity-field statistics on atomic entanglement in the Jaynes-Cummings model
}

\author{
Biplab Ghosh ${ }^{1}$, A. S. Majumdar ${ }^{2}$ and N. Nayak ${ }^{3}$ \\ S. N. Bose National Centre for Basic Sciences, Salt Lake, Kolkata 700 098, India
}

\begin{abstract}
We study the entanglement properties of a pair of two-level atoms going through a cavity one after another. The initial joint state of two successive atoms that enter the cavity is unentangled. Interactions mediated by the cavity photon field result in the final two-atom state being of a mixed entangled type. We consider the field statistics of the Fock state field, and the thermal field, respectively, inside the cavity. The entanglement of formation of the joint two-atom state is calculated for both these cases as a function of the Rabi-angle $g t$. We present a comparitive study of two-atom entanglement for low and high mean photon number cases corresponding to the different fields statistics.
\end{abstract}

\section{Introduction}

Quantum entanglement has been widely observed within the framework of quantum optical systems such as cavity quantum electrodynamics. Many beautiful experiments have been carried out in recent years and several types of entangled states have been created. Practical realization of various features of quantum entanglement are obtained in atom-photon interactions in optical and microwave cavities [1]. For implementation of quantum information protocols useful in communication and computation [2, entanglement has to be created and preserved between qubits that are well separated, and a recent experimental breakthrough has been obtained by entangling two distant atomic qubits by their interaction with the same photon 3]. From the viewpoint of information processing, quantification of entanglement is an important aspect, and recently some studies have been performed to quantify the entanglement that is obtained in atom-photon interactions in cavities [4, 5, 6, 7].

\footnotetext{
${ }^{1}$ Email: biplab@bose.res.in

${ }^{2}$ Email: archan@bose.res.in

${ }^{3}$ Email: nayak@bose.res.in
} 
In the present paper we will study the dynamical generation of entanglement between two two-level atoms mediated by various cavity fields. Since the atoms do not interact directly with each other, the properties of the radiation field encountered by them bears crucially on the nature of atomic entanglement. Our main purpose is to focus on the effect of different field dynamics on the magnitude of two-atom entanglement. The interaction between the atom and the field is governed by the Jaynes-Cummings model 8 which is experimentally realizable. The generation of nonlocal correlations between the two atoms emerging from the cavity can in general be understood using the Horodecki theorem [9], and the joint two-atom state is known to violate Bell-type inequalities [10]. Since the joint state of the two atoms emanating from the cavity is not a pure state, we quantify the entanglement using the well-known measure appropriate for mixed states, i.e., the entanglement of formation[11. We investigate how the statistics of different types of radiation fields influence the quantitative dynamics of atomic entanglement.

The structure of the paper is as follows. In Section II we review briefly the interaction between two-level atom and single mode radiation field inside a cavity described by the Jaynes-Cummings model. In Section III we show how the entanglement between two spatially separated atoms is generated. We observe robust atom-atom entanglement mediated by the Fock state field, and the thermal field, respectively. We demonstrate how the various field statistics are reflected in two-atom entanglement as a function of the average photon number of the cavity fields and the Rabi angle. Several distinctive characteristics of the entanglement generated by the different fields through the Jaynes-Cummings interaction are discussed in comparison with some earlier results obtained for the Tavis-Cummings interaction 12 . A common feature that is observed is that for the cavity low photon number case, the entanglement between the two atoms decreases with increasing average photon number of the field. A summary of our results and some concluding remarks are presented in Section IV.

\section{Entanglement mediated by the Jaynes-Cummings interaction}

The Jaynes-Cummings (JC) model is one of the most studied models in quantum optics. Our aim is to study the entanglement between atoms mediated 
by the optical field, where the light-atom interaction is governed by the JC model. The JC model conists of a two-level atom coupled to a single-mode radiation field inside a cavity. A two level atom is formally analogous to a spin- $1 / 2$ system. Let us denote the upper level of the atom as $|e\rangle$ and the lower level as $|g\rangle$ and the spin (atomic) raising and lowering operators can be defined as $\sigma^{+}=|e\rangle\langle g|$ and $\sigma^{-}=|g\rangle\langle e|$, respectively, with the commutation relation

$$
\left[\sigma^{+}, \sigma^{-}\right]=|e\rangle\langle e|-| g\rangle\langle g|=\sigma_{z} .
$$

A quantum mechanical field can be represented as (for the present purpose, we consider a single mode field)

$$
E(t)=\frac{1}{2}\left[a e^{-i \omega t}+a^{\dagger} e^{i \omega t}\right]
$$

Here $a$ and $a^{\dagger}$ are annihilation and creation operators, respectively. The graininess of the radiation field is represented by the photon number state $|n\rangle$, $n=0,1,2, \ldots .$. , such that $a|n\rangle=\sqrt{n}|n-1\rangle$ and $a^{\dagger}|n\rangle=\sqrt{n+1}|n+1\rangle$. It is an eigenstate of the number operator $\hat{n}=a^{\dagger} a$ given by $\hat{n}|n\rangle=n|n\rangle$. The field in Eq.(2) can be represented by a quantum mechanical state vector $|\psi\rangle$ which is a linear superposition of of the number states $|n\rangle$, i.e., $|\psi\rangle=\sum_{n=0}^{\infty} c_{n}|n\rangle$. where $c_{n}$ is, in general, complex and gives the probabilty of the field having $n$ photons by the relation $P_{n}=\langle n \mid \psi\rangle\langle\psi \mid n\rangle=\left|c_{n}\right|^{2}$. The field obeys quantum statistics and its average photon number is given by

$$
<n>=\sum_{n=0}^{\infty} n P_{n}
$$

with the intensity of the field $I \propto<n>$. The statistics brings in a quantum mechanical noise which is represented by the variance

$$
V=\frac{<n^{2}>-<n>}{<n^{2}>}
$$

$V=1$ is for coherent state field and $V<1$ signifies a non-classical state. The parameters $\langle n\rangle$ and $V$ give a fair description of the quantum mechanical nature of the radiation field.

The interaction picture Hamiltonian of the joint atom-field system can be written in the rotating wave approximation [13] as,

$$
H_{I}=g\left(\sigma^{+} a+\sigma^{-} a^{\dagger}\right) .
$$


where $a^{\dagger}$ and $a$ are usual creation and destruction operators of the radiation field. Here we have considered the quality factor of the cavity $Q=\infty$ since the cavity-QED related experiments are carried out with cavities with very high $Q$ [1]. We shall consider the cavity field to be in a Fock and a thermal state, respectively.

With the passage of the two atoms, one after the other, the joint state of both the atoms and the field at some instance $t$ may be denoted by $\mid \Psi(t)>_{a-a-f}$. The corresponding atom-atom-field pure density state is $\rho(t)=|\Psi(t)\rangle\langle\Psi(t)|$. In order to quantify the entanglement between the two atoms, the field variables have to be traced out. The reduced mixed density state of two atoms after taking trace over the field is

$$
\rho(t)=\operatorname{Tr} r_{\text {field }}\left(\left|\Psi(t)>_{a-a-f . a-a-f}<\Psi(t)\right|\right)
$$

Entanglement within pure states of bipartite system can be measured by the Von Neumann entropy of the reduced density matrices. For mixed states the entanglement of formation 11 is a widely used measure for computing atomic entagelement in quantum optical systems [3, 4, 5, 6, 7. 7. The entanglement of formation for a bipartite density operator $\rho$ is given by

$$
E_{F}(\rho)=h\left(\frac{1+\sqrt{1-C^{2}(\rho)}}{2}\right),
$$

where $C$ is called the concurrence defined as

$$
C(\rho)=\max \left(0, \sqrt{\lambda}_{1}-\sqrt{\lambda}_{2}-\sqrt{\lambda}_{3}-\sqrt{\lambda}_{4}\right)
$$

where the $\lambda_{i}$ are the eigenvalues of $\rho_{12}\left(\sigma_{y} \otimes \sigma_{y}\right) \rho_{12}^{*}\left(\sigma_{y} \otimes \sigma_{y}\right)$ in descending order, is the binary entropy function. The entanglement of formation is a monotone of the concurrence.

\section{Entanglement features of cavity fields}

We consider a micromaser system in which atoms are sent into the cavity at such a rate that the probability of two atoms being present there is negligibly

small. Our purpose here is to show the influence of the photon statistics of the driving fields (radiation field with which the atoms interact) on atomic entanglement. For this sake, we consider the cavity to be of a non-leaky type, 


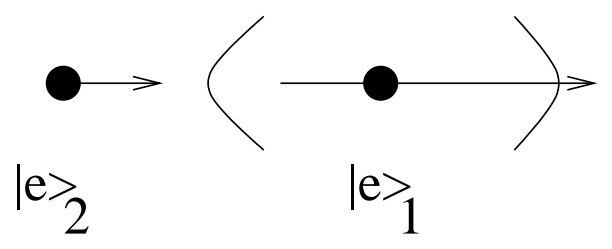

Figure 1: Two atoms prepared in excited states are pass through a single mode cavity one after the other.

that is, $Q=\infty$. In fact, the cavity-QED experiments are very close to such situations [1. In the following, we consider two different kinds of radiation fields, i.e., the Fock state field, and the thermal field, respectively.

\section{A. FOCK STATE FIELD}

A Fock state is written as $|n\rangle$ with $n$ an integer value, signifying that there are $n$ quanta of excitation in the mode. $|0\rangle$ corresponds to the ground state (no excitation). Fock states form the most convenient basis of the Fock space. The amplitudes $c_{n}$ obey the delta function relation $c_{n}=\delta_{m, n}$ where $m$ is the photon number of the Fock state. The variance is given by $V=1-\frac{1}{m}$. So, for small values of $m, V<1$ and field has non-classical character. For large $m, V$ tends towards the classical limit. This feature is reflected in the entanglement generated between the two atoms, as we shall see later.

Let us first consider the passage of the first atom, initially in the excited state $\mid e>$, through the cavity. The joint atom-field state is given by

$$
|\Psi(t=0)\rangle_{a-f}=|e\rangle \otimes|n\rangle .
$$

The atom-field wave function evolves with the interaction given by Eq.(5) to

$$
\left|\Psi(t)_{a-f}\right\rangle=\cos (\sqrt{n+1} g t)|e, n\rangle+\sin (\sqrt{n+1} g t)|g, n+1\rangle
$$

The next atom which enters the cavity interacts with this "changed" field and thus a correlation develops between the two atoms via the cavity field. The joint state of the two atoms and the field is given by

$$
|\Psi(t)\rangle_{a-a-f}=\alpha_{1}\left|e_{1}, e_{2}, n\right\rangle+\alpha_{2}\left|e_{1}, g_{2}, n+1\right\rangle+\alpha_{3}\left|g_{1}, e_{2}, n+1\right\rangle+\alpha_{4}\left|g_{1}, g_{2}, n+2\right\rangle(11)
$$


where

$$
\begin{array}{r}
\alpha_{1}=\cos ^{2}(\sqrt{n+1} g t), \quad \alpha_{2}=\cos (\sqrt{n+1} g t) \sin (\sqrt{n+1} g t), \\
\alpha_{3}=\cos (\sqrt{n+2} g t) \sin (\sqrt{n+1} g t), \quad \alpha_{4}=\sin (\sqrt{n+1} g t) \sin (\sqrt{n+2} g t) .
\end{array}
$$

The reduced mixed density state of two atoms after tracing over the field is given by (we display the non-vanishing terms only)

$$
\begin{array}{r}
\rho(t)_{a-a}=\operatorname{tr}_{f}\left(\rho(t)_{a-a-f}\right)=\alpha_{1}^{2}\left|e_{1} e_{2}\right\rangle\left\langle e_{1} e_{2}\left|+\alpha_{2}^{2}\right| e_{1} g_{2}\right\rangle\left\langle e_{1} g_{2}\right| \\
+\alpha_{3}^{2}\left|g_{1} e_{2}\right\rangle\left\langle g_{1} e_{2}\left|+\alpha_{2} \alpha_{3}\right| e_{1} g_{2}\right\rangle\left\langle g_{1} e_{2}\left|+\alpha_{2} \alpha_{3}\right| g_{1} e_{2}\right\rangle\left\langle e_{1} g_{2}\left|+\alpha_{4}^{2}\right| g_{1} g_{2}\right\rangle\left\langle g_{1} g_{2}\right| .
\end{array}
$$

We compute the entanglement of formation $E_{f}$ for this bipartite two-atom state. In Figure $2 E_{F}$ is plotted versus the Rabi angle $g t$ for different values of $n$.

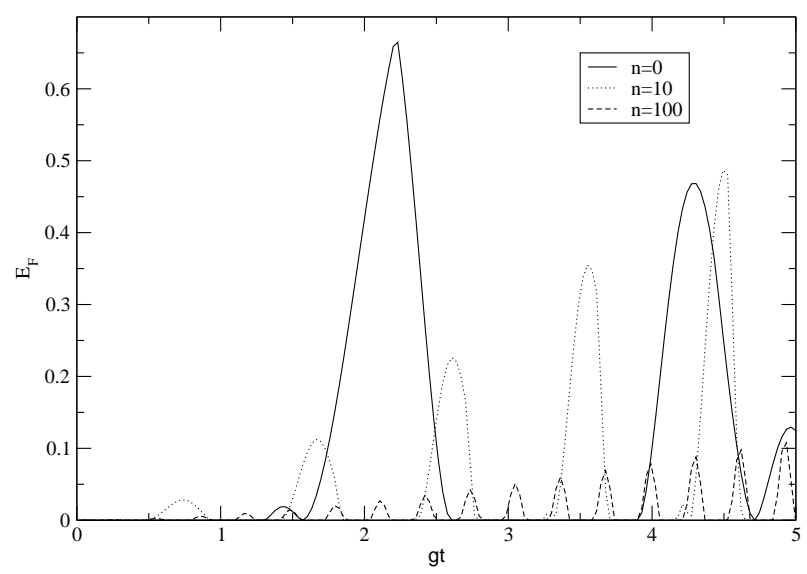

Figure 2: Atom-atom entanglement versus $g t$. Solid line, dotted line, and dashed line indicate $E_{F}$ between two atoms when the cavity fock states are $n=0, n=10$, and $n=100$ respectively.

The peaks of the entanglement of formation are reflective of the photon statistics that are typical in micromaser dynamics [14]. We see that $E_{F}$ falls off sharply as $n$ increases. The non-classical character of the field for small values of the average photon number $n$, is reflected in larger entanglement between the two atoms. An interesting comparison can be made with the 
case of the Tavis-Cummings model[12] which is employed when two atoms are present simultaneously inside the cavity. Although simultaneous interaction of two excited atoms with Fock state field never results in two-atom entanglement as was shown by Tessier et al. [7], the notable difference here is that in the $\mathrm{JC}$ dynamics modelling the micromaser one always gets two-atom entanglement mediated by the Fock state cavity field, as we see in Figure 2.

\section{B. THERMAL FIELD}

The thermal field is the most easily available radiation field, and so, its influence on the entanglement of spins is of much interest. The field at thermal equilibrium obeying Bose-Einstein statistics has an average photon number at temperature $T^{0} K$, given by

$$
<n>=\frac{1}{e^{\hbar \omega / k T}-1} \text {. }
$$

The photon statistics is governed by the distribution $P_{n}$ given by

$$
P_{n}=\frac{<n>^{n}}{(1+<n>)^{n+1}} .
$$

This distribution function always peaks at zero, i.e., $n_{\text {peak }}=0$. For a thermal field distribution function for the cavity field, the joint two-atom-cavity state is obtained by summing over all $n$, and is given by

$$
\begin{aligned}
& |\Psi(t)\rangle_{a-a-f}=\sum_{n} A_{n}\left[\cos ^{2}(\sqrt{n+1} g t)\left|e_{1}, e_{2}, n\right\rangle+\cos (\sqrt{n+1} g t) \sin (\sqrt{n+1} g t)\left|e_{1}, g_{2}, n+1\right\rangle\right. \\
& \left.+\cos (\sqrt{n+2} g t) \sin (\sqrt{n+1} g t)\left|g_{1}, e_{2}, n+1\right\rangle+\sin (\sqrt{n+1} g t) \sin (\sqrt{n+2} g t)\left|g_{1}, g_{2}, n+2\right\rangle\right](16)
\end{aligned}
$$

where $P_{n}=\left|A_{n}\right|^{2}$ is the photon distribution function of the thermal field.

The reduced mixed density state of two atoms after passing through the the thermal cavity field can be written as

$$
\begin{array}{r}
\rho(t)_{a-a}=\operatorname{tr}_{f}\left(\rho(t)_{a-a-f}\right)=\beta_{1}\left|e_{1} e_{2}\right\rangle\left\langle e_{1} e_{2}\left|+\beta_{2}\right| e_{1} g_{2}\right\rangle\left\langle e_{1} g_{2}\right| \\
+\beta_{3}\left|g_{1} e_{2}\right\rangle\left\langle g_{1} e_{2}\left|+\beta_{4}\right| e_{1} g_{2}\right\rangle\left\langle g_{1} e_{2}\left|+\beta_{4}\right| g_{1} e_{2}\right\rangle\left\langle e_{1} g_{2}\left|+\beta_{5}\right| g_{1} g_{2}\right\rangle\left\langle g_{1} g_{2}\right|,
\end{array}
$$

where

$$
\beta_{1}=\sum_{n} P_{n} \cos ^{4}(\sqrt{n+1} g t), \beta_{2}=\sum_{n} P_{n} \cos ^{2}(\sqrt{n+1} g t) \times \sin ^{2}(\sqrt{n+1} g t),
$$




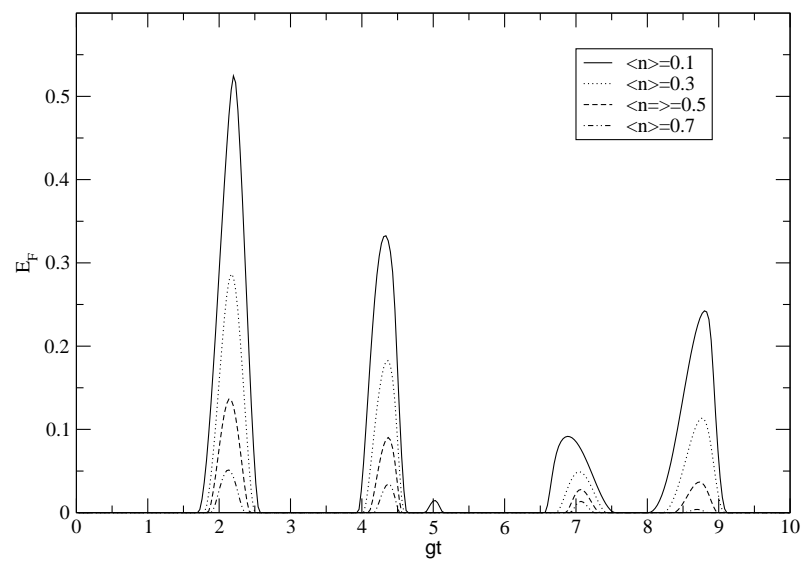

Figure 3: Atom-atom entanglement of formation mediated by the thermal cavity field is plotted versus $g t$.

$$
\begin{gathered}
\beta_{3}=\sum_{n} P_{n} \cos ^{2}(\sqrt{n+2} g t) \times \sin ^{2}(\sqrt{n+1} g t), \beta_{5}=\sum_{n} P_{n} \sin ^{2}(\sqrt{n+1} g t) \times \sin ^{2}(\sqrt{n+2} g t), \\
\beta_{4}=\sum_{n} P_{n} \sin ^{2}(\sqrt{n+1} g t) \times \cos (\sqrt{n+1} g t) \cos (\sqrt{n+2} g t) .
\end{gathered}
$$

We compute the entanglement of formation $E_{F}$ for the above two-atom state and plot it versus the Rabi angle $g t$ for different values of average photon number $\langle n\rangle$ in Figure 3. It is interesting to note that the thermal field which has miminimal information can nevertheless entangle qubits that are prepared initially in a separable state. In the context of the Tavis-Cummings framework when both the atoms interact simultaneously with the radiation field, Kim et al. [6] have noticed similar trends in the entanglement mediated by the thermal field. Thus both Jaynes-Cummings and the Tavis-Cummings models of atom-photon interaction generate similar entanglement when the radiation field is thermal, whereas for the Fock state field case the situation is contrasting as observed earlier.

\section{Conclusions}

To summarize, in this paper we have presented a realistic micromaser-type model where two spatially separated atoms are entangled via a cavity field. 
The entanglement between the two separate atoms builds up via atom-photon interactions inside the cavity, even though no single atom interacts directly with another. We have computed the two-atom entanglement as measured by the entanglement of formation $E_{F}$, for the case of two different types of radiation fields, i.e., the Fock state field and the thermal field, respectively. Our purpose has been to study the effects of the statistics of the bosonic radiation field on the dynamics of the entanglement of two atomic qubits, i.e., two fermionic systems. Several interesting features of atomic entanglement are observed.

We first show that for the Fock state cavity field, entanglement between two successively passing atoms can be generated as a consequence of JaynesCummings (JC) dynamics. This is in contrast to the case when both the atoms reside together inside the cavity when Tavis-Cummings (TC) dynamics for atom-photon interactions is unable to generate atomic entanglement [7. We then study the entanglement mediated by the thermal radiation field. It is interesting to note that the thermal field which carries minimum information is still able to produce atomic entanglement through both the JC interaction as seen here, and also through the TC interaction as was observed earlier 6 . However, the thermal field having a high value of the average photon number loses its ability to entangle atomic qubits passing through it.

Finally, we would like to reemphasize that the quantitative study of entanglement produced in various types of atom-photon interactions is a relevant arena for investigations. Atom-photon interactions and the generation of entanglement mediated through them are expected to play an important role in possible future practical realizatons in the field of quantum communications [3, 15. Recently, the possibility of entanglement of a thermal radiation field with high temperature phonons associated with moving mirrors of a cavity has been shown[16, brightening the prospects for creating macroscopic entanglement. Even from a purely pedagogical perspective, investigations of quantitative entanglement in atom-photon interactions could

lead to interesting insights on the curious properties of entanglement such as its 'monogamous' nature[17.

\section{References}

[1] J. M. Raimond, M. Brune and S. Haroche, Rev. Mod. Phys. 73, 565 (2001). 
[2] See, for example, M. A. Nielsen and I. L. Chuang, Quantum Computation and Information (Cambridge University Press, Cambridge, England, 2000).

[3] D. N. Matsukevich, T. Chanelire, S. D. Jenkins, S.-Y. Lan, T. A. B. Kennedy, and A. Kuzmich, Phys. Rev. Lett. 96, 030405 (2006).

[4] P. Masiak, Phys. Rev. A 66, 023804 (2002).

[5] A. Datta, B. Ghosh, A. S. Majumdar and N. Nayak, Europhys. Lett. 67, 934 (2004).

[6] M. S. Kim, Jinhyoung Lee, D. Ahn and P. L. Knight, Phys. Rev. A 65, 040101(R) (2002); L. Zhou, H. S. Song and C. Li, J. Opt. B: Quantum Semiclass. Opt. 4, 425 (2002).

[7] T. Tessier, A. Delgado, I. Fuentes-Guridi, and I. H. Deutsch, Phys. Rev.A 68, 062316 (2003).

[8] E. T. Jaynes, F. W. Cummings, Proc. IEEE 51, 89 (1963).

[9] Horodecki et al., Phys. Lett. A, 200, 340 (1995).

[10] A. S. Majumdar and N. Nayak, Phys. Rev. A64, 013821 (2001).

[11] S. Hill and W. K. Wootters, Phys. Rev. Lett. 78, 5022 (1997); W. K. Wootters, Phys. Rev. Lett. 802245 (1998).

[12] M. Tavis and F. W. Cummings, Phys. Rev. 170, 379 (1968).

[13] See, for instance, W. H. Louisell, Quantum Statistical Properties of Radiation (Wiley, New York, 1973).

[14] N. Nayak, A. S. Majumdar and V. Bartzis, Nonlinear Optics 24, 319 (2000).

[15] J. Volz, M. Weber, D. Schlenk, W. Rosenfeld, J. Vrana, K. Saucke, C. Kurtsiefer, and H. Weinfurter, Phys. Rev. Lett. 96, 030404 (2006).

[16] A. Ferreira, A. Guerreiro, and V. Vedral, Phys. Rev. Lett. 96, 060407 (2006).

[17] B. Ghosh, A. S. Majumdar, and N. Nayak, quant-ph/0505037 (to appear in Int. J. Quant. Inf.). 